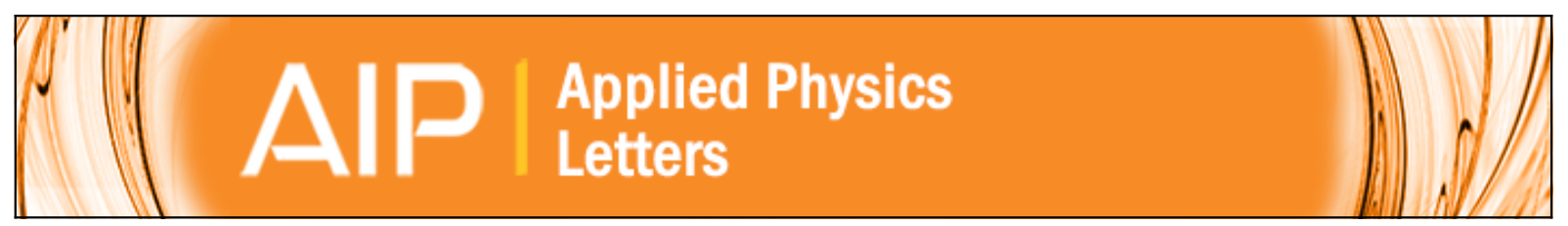

\title{
Optically addressed near and long-wave infrared multiband photodetectors
}

O. O. Cellek, J. L. Reno, and Y.-H. Zhang

Citation: Applied Physics Letters 100, 241103 (2012); doi: 10.1063/1.4729004

View online: http://dx.doi.org/10.1063/1.4729004

View Table of Contents: http://scitation.aip.org/content/aip/journal/apl/100/24?ver=pdfcov

Published by the AIP Publishing

\section{Articles you may be interested in}

Optically-aligned visible/near-infrared dual-band photodetector materials and devices on GaAs using metamorphic epitaxy

J. Appl. Phys. 110, 063109 (2011); 10.1063/1.3642981

Binary superlattice quantum-well infrared photodetectors for long-wavelength broadband detection

Appl. Phys. Lett. 84, 5127 (2004); 10.1063/1.1764932

Quantum-well infrared photodetectors with digital graded superlattice barrier for long-wavelength and broadband detection

Appl. Phys. Lett. 75, 3207 (1999); 10.1063/1.125279

Optical coupling in quantum well infrared photodetector by diffraction grating

J. Appl. Phys. 84, 5750 (1998); 10.1063/1.368840

Resonant-cavity InGaAs/InAIGaAs/InP photodetector arrays for wavelength demultiplexing applications Appl. Phys. Lett. 70, 2347 (1997); 10.1063/1.118870 


\title{
Optically addressed near and long-wave infrared multiband photodetectors
}

\author{
O. O. Cellek, ${ }^{1}$ J. L. Reno, ${ }^{2}$ and Y.-H. Zhang ${ }^{1, a)}$ \\ ${ }^{1}$ Center for Photonics Innovation and School of Electrical, Computer, and Energy Engineering, \\ Arizona State University, Tempe, Arizona 85287, USA \\ ${ }^{2}$ Center for Integrated Nanotechnologies, Sandia National Laboratories, Albuquerque, New Mexico 87185 , \\ USA
}

(Received 11 April 2012; accepted 24 May 2012; published online 11 June 2012)

Optically addressed dual-band photodetector incorporating of a $0.82 \mu \mathrm{m}$ cut-off wavelength near-infrared (NIR) AlGaAs/GaAs p-i-n photodetector and a $8.2 \mu \mathrm{m}$ peak wavelength long-wave infrared (LWIR) $\mathrm{AlGaAs} / \mathrm{GaAs}$ quantum well infrared photodetector on GaAs substrate is fabricated and characterized. Switching between NIR and LWIR bands is demonstrated by using external light bias. The dual-band photodetector gives 65\% quantum efficiency in NIR band and specific detectivity of $2 \times 10^{9} \mathrm{~cm} \mathrm{~Hz}^{1 / 2} / \mathrm{W}$ in LWIR band at $68 \mathrm{~K}$. Spectral crosstalk is better than $25 \mathrm{~dB}$. These devices enable the use of only a single indium-bump per pixel for multiband image sensor arrays to have maximum fill factor. (C) 2012 American Institute of Physics. [http://dx.doi.org/10.1063/1.4729004]

Mid-wave infrared band (MWIR, 3-5 $\mu \mathrm{m}$ ) and long-wave infrared band (LWIR, 8-12 $\mu \mathrm{m}$ ) photodetectors and their focal plane arrays (FPA) are important for chemical sensing, environmental monitoring, natural resources survey, and defense applications. Adding near infrared (NIR, 0.75-1.1 $\mu \mathrm{m}$ ) and short-wave infrared (SWIR, 1.1-2.5 $\mu \mathrm{m}$ ) band detection capability to them allows new applications.

Band switching and spectral tuning by electrical bias polarity switching and voltage tuning have been demonstrated with $\mathrm{HgCdTe}$ and quantum well infrared photodetectors (QWIP), respectively. ${ }^{1,2}$ Multiband infrared FPA using these principles or using more than two terminals per pixel have been reported. ${ }^{2-9}$ Dual-band type-II superlattice infrared photodetectors, ${ }^{10,11}$ fourcolor QWIPs, ${ }^{12}$ voltage-tunable quantum-dot infrared photodetectors (QDIP), ${ }^{13}$ and voltage-switchable NIR/LWIR imaging with QWIPs were also reported. ${ }^{14}$ Without band switching, InSb, HgCdTe, QWIP, ${ }^{15-17}$ and InGaAs (Ref. 18) detectors can detect light with broad band from visible/NIR to MWIR/LWIR ranges. Theory of voltage-tuning in multi-color QWIP stacks has been discussed in the literature. ${ }^{19,20}$ However, none of the demonstrated technologies were able to switch bands with externally applied light in the infrared.

Optically addressed multiband detectors ${ }^{21,22}$ can switch detection bands by external optical bias and can be fabricated by using two terminals (single indium-bump per pixel), which maximizes the pixel fill factor in large infrared (IR) FPAs using small pixel pitch. It also simplifies the read-out integrated circuit (ROIC) for multiband FPA. Furthermore, when utilized with existing multiband detector technologies, it enables adding at least one more extra detection band without the need to change the ROIC design. The proposed architecture also allows low spectral crosstalk, high band rejection ratio, and, in principle, can have an infinite number of bands.

The preliminary proof of concept has been recently demonstrated using a triple-junction photodetector covering visible, NIR, and SWIR bands..$^{21}$ The photodetector consisted of a set of p-n junction sub-photodetectors with different cutoff

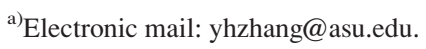

wavelengths and series connected through tunnel junctions. Incident photons pass through all the sub-photodetectors whose bandgaps have higher energies than the photons' and are absorbed by the corresponding sub-photodetector. All subphotodetectors except one are illuminated by a series of light emitting diodes (LED) with their corresponding detection wavelengths, and the non-illuminated sub-photodetector remains the only active photodetector since it limits the total current. In order to extend this idea into technologically important NIR and LWIR bands, we use unipolar and bipolar devices to eliminate the need for the tunnel junctions. Equivalent circuit modeling of this design showed that NIR/LWIR band switching is indeed feasible using the optical addressing technique. ${ }^{23}$

In this paper, we experimentally demonstrate the NIR/LWIR dual-band optically addressed photodetector and characterize its performance. The photodetector consisted of two sub-photodetectors. For the LWIR band detection, a AlGaAs/GaAs QWIP is used as first sub-photodetector, and for the NIR band detection, a GaAs p-i-n photodetector is grown on the QWIP as the second sub-photodetector (Sample Reference: VA0411). The optical bias source for optical addressing is placed outside of the signal beam path (Fig. 1). The photodetector is defined by mesa etching and connected with conventional ohmic contacts. The entire device is kept under constant electrical bias such that mesa top is negative and $\mathrm{p}$-i-n sub-photodetector is reverse biased. The total current is limited by the higher resistance NIR p-i-n subphotodetector without any optical bias, because the two sub-photodetectors are series connected. For front-side illumination, when the NIR p-i-n sub-photodetector is grown on top of the LWIR sub-photodetector, the optical bias source illuminates the $\mathrm{p}-\mathrm{i}-\mathrm{n}$ layers first and decreases its resistance. Since both sub-photodetectors are series connected, when the NIR optical bias is on, the photodetector current is limited by the smaller current of QWIP sub-photodetector. As the NIR optical bias photon flux is increased, the p-i-n subphotodetector impedance can be reduced to be orders of magnitude lower than that of the QWIP. The output photocurrent can be written as ${ }^{23}$ 


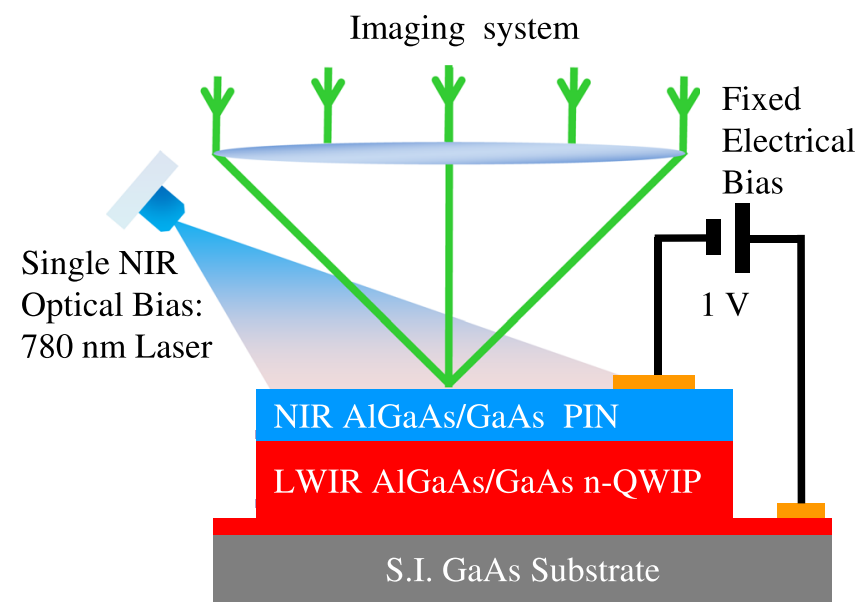

FIG. 1. Schematic of NIR/LWIR optically addressed photodetector using single optical bias source.

$$
i_{\text {out }}=i_{Q W I P} \frac{r_{Q W I P}}{r_{Q W I P}+r_{P I N}}+i_{P I N} \frac{r_{P I N}}{r_{Q W I P}+r_{P I N}},
$$

where $i_{Q W I P}$ and $i_{P I N}$ are the AC-currents and $r_{Q W I P}$ and $r_{P I N}$ are the AC-resistances of the QWIP and the p-i-n subphotodetector, respectively. Without optical bias, $r_{\text {OWIP }} \ll r_{P I N}$ holds and the output current is dominated by $\mathrm{p}-\mathrm{i}-\mathrm{n}$ subphotodetector photocurrent, and the device is in the NIR mode of operation. When optical bias is applied onto the p-i-n subphotodetector, this changes to $r_{Q W I P} \gg r_{P I N}$ and the output becomes dominated by QWIP sub-photodetector photocurrent and the device is in the LWIR mode of operation.

The device structure used for this study is grown with molecular beam epitaxy on (100) GaAs substrate. The QWIP sub-photodetector structure consists of a $1000 \mathrm{~nm}$ thick $10^{18} \mathrm{~cm}^{-3} \mathrm{Si}$ doped n-type GaAs layer for a bottom contact and an absorbing layer consisting of 30 periods of $4.6 \mathrm{~nm}$ GaAs quantum wells with $50 \mathrm{~nm} \mathrm{Al}_{0.27} \mathrm{Ga}_{0.73} \mathrm{As}$ barriers. The center $4.0 \mathrm{~nm}$ regions in the quantum wells are n-doped with Si at $4 \times 10^{17} \mathrm{~cm}^{-3}$. On top of the QWIP structure, the layers of the p-i-n sub-photodetector are grown in following sequence: $500 \mathrm{~nm} \mathrm{n-GaAs} \mathrm{doped} \mathrm{with} \mathrm{Si}$ at $10^{18} \mathrm{~cm}^{-3}$, $2500 \mathrm{~nm}$ n-GaAs doped with Si at $2 \times 10^{16} \mathrm{~cm}^{-3}$, and $400 \mathrm{~nm}$ $\mathrm{p}-\mathrm{Al}_{0.27} \mathrm{Ga}_{0.73} \mathrm{As}$ window layer doped with $\mathrm{C}$ at $10^{18} \mathrm{~cm}^{-3}$ concentration. The cap layer is a $10 \mathrm{~nm}$ p-GaAs doped with $\mathrm{C}$ at $1.4 \times 10^{18} \mathrm{~cm}^{-3}$. The device mesa size $150 \mu \mathrm{m} \times 150 \mu \mathrm{m}$ is defined by wet chemical etching. The packaged photodetector is mounted in a cryostat with a $\mathrm{ZnSe}$ front window and a quartz side window. The $\mathrm{ZnSe}$ front window is used for normal incidence optical response characterization, while the quartz window is used for the optical biasing light.

The I-V curves plotted in Fig. 2 are obtained at different detector temperatures without any optical bias and with $0.12 \mathrm{~W} / \mathrm{cm}^{2}$ optical bias at $780 \mathrm{~nm}$. With an applied optical bias the device is in LWIR mode of operation where the QWIP is the current limiting sub-photodetector. A typical AlGaAs/GaAs QWIP I-V curve ${ }^{24}$ is observed and showed current saturation under voltage bias from -1 to $-3 \mathrm{~V}$. Open circuit voltage of the $\mathrm{p}$-i-n sub-photodetector under optical bias shifts the zero crossing of the I-V curve by $1.4 \mathrm{~V}$ to positive bias region. As the temperature reaches $90 \mathrm{~K}$, the dark current of the QWIP sub-photodetector increases so that the

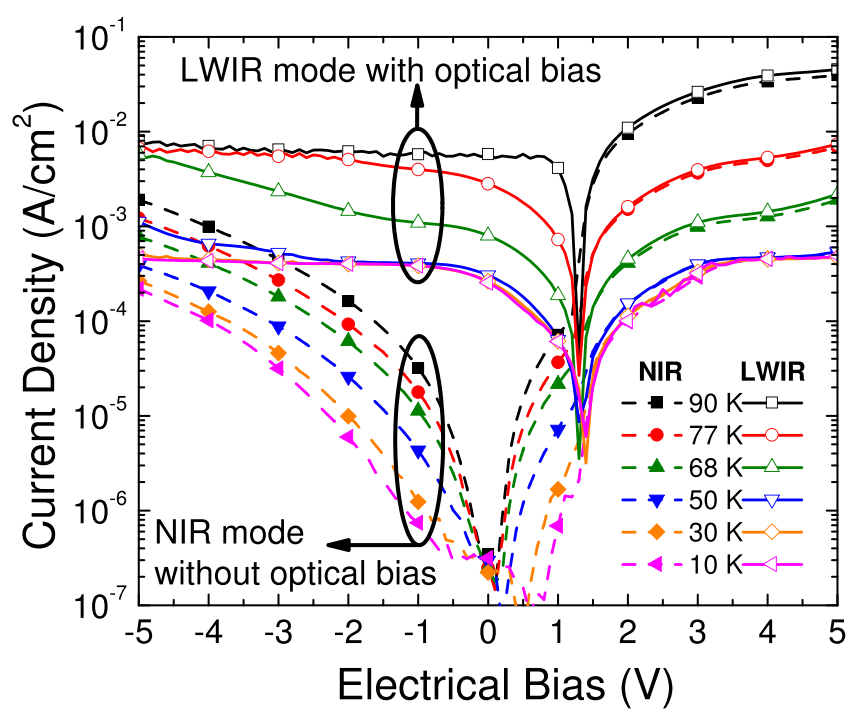

FIG. 2. Absolute value of the current density vs. electrical bias measured in a dewar with $180^{\circ}$ field of view at temperatures $10,30,50,68,77$, and $90 \mathrm{~K}$. While the detector is in NIR mode, the p-i-n photodetector determines the current, while the detector is in LWIR mode the QWIP determines the current.

p-i-n sub-photodetector under optical bias becomes the current limiting device. Without optical bias, the photodetector is in NIR mode of operation, and typical NIR p-i-n photodiode characteristics is observed in reverse bias with leakage current on the order of $10^{-6} \mathrm{~A} / \mathrm{cm}^{2}$. In forward bias, the $\mathrm{p}-\mathrm{i}-\mathrm{n}$ sub-photodetector turns on at $1.4 \mathrm{~V}$, and then the QWIP subphotodetector becomes the current limiting device. Under higher bias voltages, the current density with optical bias follows the current density without optical bias within a band of less than $10 \%$ increase. This increase in QWIP current is due to heating of the pixel with the bias laser light.

NIR responsivity is measured with a $780 \mathrm{~nm}$ LED. LWIR responsivity is measured under optical bias from a $780 \mathrm{~nm}$ laser diode and a chopped blackbody source with an IR filter. The output photocurrent signal is amplified with a current preamplifier connected to a lock-in amplifier. While the photodetector is kept at constant bias of $-1 \mathrm{~V}$ (mesa top negative), NIR, and LWIR responsivity is measured under different optical bias levels (Fig. 3). The NIR/LWIR band switching threshold occurs at optical bias power interval of $1-10 \mathrm{~mW} / \mathrm{cm}^{2}$ at $68 \mathrm{~K}$. As the operating temperature increases, the increased QWIP dark current requires a higher optical bias threshold to switch bands. Figure 3 shows that LWIR peak responsivity is $75 \mathrm{~mA} / \mathrm{W}$ and remains constant for optical bias down to $6 \mathrm{~mW} / \mathrm{cm}^{2}$. The LWIR signal was too weak to be measured at an optical bias below $6 \mathrm{~mW} / \mathrm{cm}^{2}$. The result proves that the LWIR band can be switched off effectively by decreasing the optical bias below the optical bias threshold. The measured peak responsivity corresponds to a LWIR quantum efficiency of $1 \%$, in good agreement with the typical value for this frontilluminated normal incidence QWIPs without grating coupling. ${ }^{24}$ Figure 3 shows that NIR peak responsivity at the cut-off wavelength is constant at $0.43 \mathrm{~A} / \mathrm{W}$ and the NIR photodetector is operational with $\mathrm{QE}$ of $65 \%$ under optical biases below $3 \mathrm{~mW} / \mathrm{cm}^{2}$. At optical biases higher than $6 \mathrm{~mW} / \mathrm{cm}^{2}$, the device operates in the LWIR mode with a measured NIR responsivity of $2.7 \mathrm{~mA} / \mathrm{W}$. The NIR band rejection ratio (BRR) at the cutoff wavelength can be defined as 


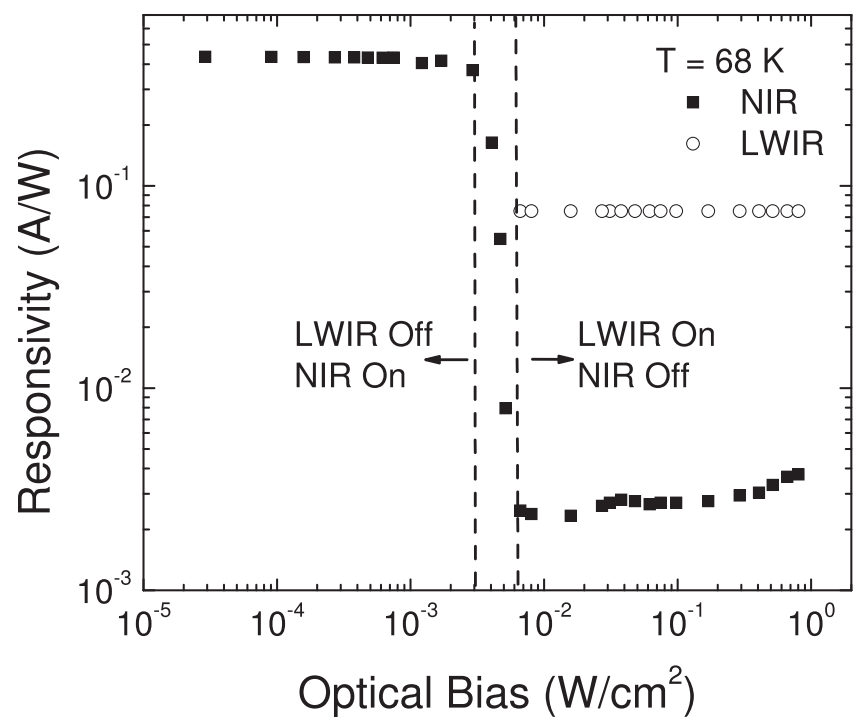

FIG. 3. Peak responsivity vs. applied optical bias at $68 \mathrm{~K}$. Band switching between NIR and LWIR occurs at $3-6 \mathrm{~mW} / \mathrm{cm}^{2}$ optical bias at $780 \mathrm{~nm}$ wavelength.

$$
B R R_{N I R}=10 \log \frac{R_{N I R}^{N I R}}{R_{N I R}^{L W I R}}=10 \log \frac{0.43 \mathrm{~A} / \mathrm{W}}{2.7 \mathrm{~mA} / \mathrm{W}}=22 \mathrm{~dB}
$$

where $R_{N I R}^{N I R}$ and $R_{N I R}^{L W I R}$ are peak NIR responsivities (A/W units) in NIR and LWIR mode of operation, respectively. The LWIR band rejection is higher than the measurement limit which is determined by the $54 \mathrm{~dB}$ dynamic range of the lock-in amplifier (Stanford Research Systems SR830) and under the measurement conditions $B_{R R} R_{L I R}>30 \mathrm{~dB}$. The spectral crosstalk between the bands can be written using the same notation in the literature. ${ }^{25}$ Assuming a scenario of constant irradiance in all wavelengths, crosstalk (CT) of NIR on LWIR can be written as

$$
C T_{L W I R}^{N I R}=10 \log \frac{\int_{0}^{\infty} R_{L W I R}(\lambda)}{\int_{0}^{1.1 \mu \mathrm{m}} R_{L W I R}(\lambda)} \sim 25 \mathrm{~dB},
$$

where $R_{\text {LWIR }}(\lambda)$ is spectral responsivity of the device in LWIR mode. The numerator is the integrated photocurrent in both bands, while the denominator is the integrated photocurrent in the NIR band only. Crosstalk of LWIR on NIR is also limited by the measurement set-up and $C T_{N I R}^{L W I R}>30 \mathrm{~dB}$.

Spectral response of the photodetector is measured with an FTIR system equipped with $\mathrm{KBr}$ and quartz beam splitters which are installed separately for the measurements in LWIR and NIR bands, respectively. Calibrated Si and MCT detectors are used with a halogen lamp as visible and a globar as infrared light sources. Under $1 \mathrm{~W} / \mathrm{cm}^{2} 780 \mathrm{~nm}$ optical bias and at $77 \mathrm{~K}$, the LWIR spectrum is measured, and the peak wavelength of the QWIP is $8.2 \mu \mathrm{m}$ with a full width at half maximum of $1.4 \mu \mathrm{m}$ (Fig. 4). NIR spectrum is measured without optical bias, and the cut-off wavelength is at $820 \mathrm{~nm}$. Visible response of the photodetector is strongly attenuated due to the thick AlGaAs window layer.

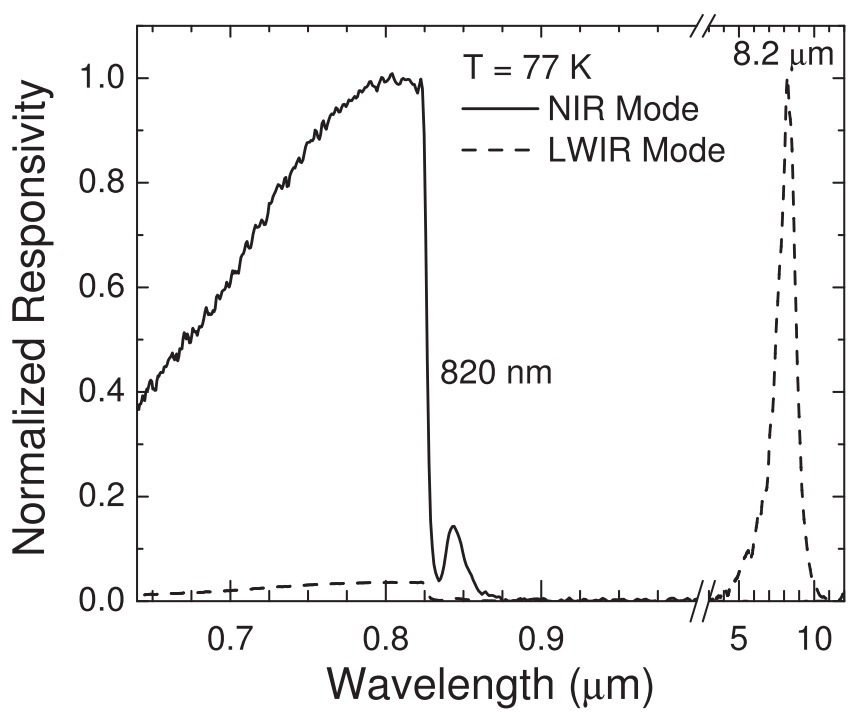

FIG. 4. Spectral response in NIR and LWIR modes of operation at $77 \mathrm{~K}$ measured with different beam splitters in FTIR.

Peak specific detectivity $\left(D^{*}\right)$ of the detector in LWIR mode is measured to be $2 \times 10^{9} \mathrm{~cm} \mathrm{~Hz}^{1 / 2} / \mathrm{W}$ under $290 \mathrm{~K}$ background and $180^{\circ}$ field of view, at an optical bias of $0.12 \mathrm{~W} / \mathrm{cm}^{2}$ and an electrical bias of $-1 \mathrm{~V}$. The detectivity will improve with grating coupling, the same QWIP design with back-side illumination and top-side etched grating coupling is suitable for FPA imaging with long integration times. ${ }^{6,24,26}$ Figure 5 shows the peak responsivity and $\mathrm{D}^{*}$ vs. bias voltage for the LWIR mode of operation. Measurements of the LWIR D* under different electrical biases show that the peak $D^{*}$ decreases as the electrical bias increases due to an increase in QWIP dark current. LWIR responsivity has a negative differential slope on -1 to $-2 \mathrm{~V}$ electrical bias range, consistent with early reports and related with intervalley transfer of electrons. ${ }^{24,27,28}$ The result shows that the p-i-n sub-photodetector does not affect the transport characteristics of the QWIP under the reported operating conditions, and the LWIR QWIP signal is as high as its single band counterparts. ${ }^{24}$ In NIR mode, photodetector noise is limited by the system noise which is above the shot noise limit due to low current density, on the order of $10^{-6} \mathrm{~A} / \mathrm{cm}^{2}$ at $68 \mathrm{~K}$.

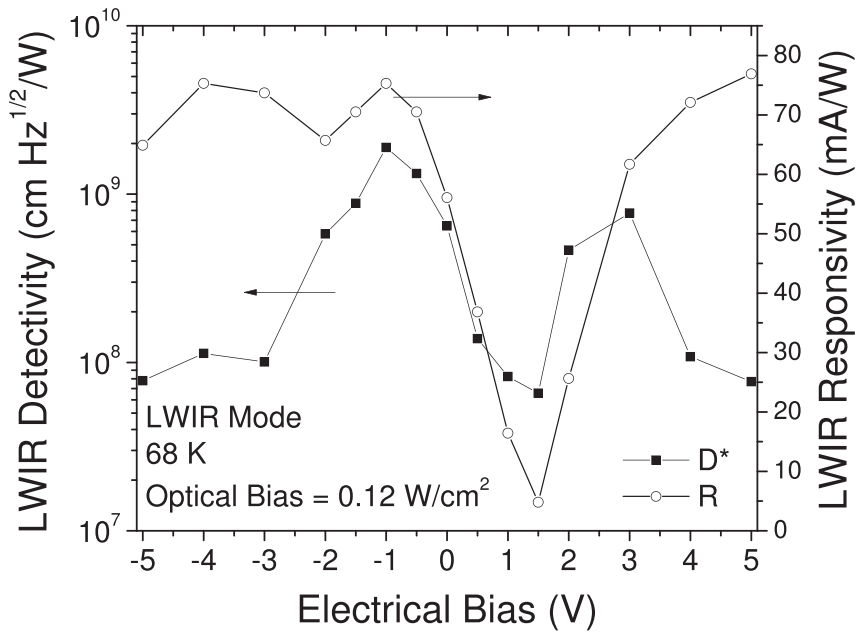

FIG. 5. Detectivity and responsivity vs. applied bias in LWIR band at $780 \mathrm{~nm}$ optical bias of $0.12 \mathrm{~W} / \mathrm{cm}^{2}$. 
In summary, NIR/LWIR optically addressed dual band infrared photodetector has been experimentally demonstrated. The architecture allows single-indium bump per pixel infrared FPAs, and using a bipolar NIR sub-photodetector with a unipolar LWIR sub-photodetector eliminated the need for tunnel junctions. Due to large impedance difference between NIR and LWIR sub-photodetectors, only a single optical bias source is needed for dual-band switching. Further addition of more detection bands is possible by using different active layers grown on the same substrate and by adding more corresponding optical bias sources. Measured LWIR D* value shows that the device is suitable for passive imaging in LWIR band.

This work was performed, in part, at the Center for Integrated Nanotechnologies, a U.S. Department of Energy, Office of Basic Energy Sciences user facility. Sandia National Laboratories is a multi-program laboratory managed and operated by Sandia Corporation, a wholly owned subsidiary of Lockheed Martin Corporation, for the U.S. Department of Energy's National Nuclear Security Administration under contract DE-AC04-94AL85000. O.O.C. and Y.H.Z. also acknowledge funding by the AFOSR under Grant No. FA9550-10-1-0129.

${ }^{1}$ I. Gravé, A. Shakouri, N. Kuze, and A. Yariv, Appl. Phys. Lett. 60, 2362 (1992).

${ }^{2}$ E. R. Blazejewski, J. M. Arias, G. M. Williams, W. McLevige, M. Zandian, and J. Pasko, J. Vac. Sci. Tech. B 10, 1626 (1992).

${ }^{3}$ E. P. G. Smith, L. T. Pham, G. M. Venzor, E. M. Norton, M. D. Newton, P. M. Goetz, V. K. Randall, A. M. Gallagher, G. K. Pierce, E. A. Patten, R. A. Coussa, K. Kosai, W. A. Radford, L. M. Giegerıch, J. M. Edwards, S. M. Johnson, S. T. Baur, J. A. Roth, B. Nosho, T. J. De Lyon, J. E. Jensen, and R. E. Longshore, J. Electron. Mater. 33, 509 (2004).

${ }^{4}$ P. Abbott, L. Pillans, P. Knowles, and R. K. M. Ewen, Proc. SPIE 7660, 766035 (2010).

${ }^{5}$ M. Sundaram, S. C. Wang, M. F. Taylor, A. Reisinger, G. L. Milne, K. B. Reiff, R. E. Rose, and R. R. Martin, Infrared Phys. Technol. 42, 301 (2001).
${ }^{6}$ S. D. Gunapala, S. V. Bandara, J. K. Liu, J. M. Mumolo, D. Z. Ting, C. J. Hill, J. Nguyen, B. Simolon, J. Woolaway, S. C. Wang, W. Li, P. D. LeVan, and M. Z. Tidrow, IEEE J. Quantum Electron. 46, 285 (2010).

${ }^{7}$ A. Majumdar, K. K. Choi, J. L. Reno, and D. C. Tsui, Appl. Phys. Lett. 86, 261110 (2005).

${ }^{8}$ S. U. Eker, M. Kaldirim, Y. Arslan, and C. Besikci, IEEE Electron Device Lett. 29, 1121 (2008).

${ }^{9}$ A. Walther, R. Rehm, J. Fleissner, J. Schmitz, J. Ziegler, W. Cabanski, and R. Breiter, Proc. SPIE 6542, 654206 (2007).

${ }^{10}$ A. Khoshakhlagh, J. B. Rodriguez, E. Plis, G. D. Bishop, Y. D. Sharma, H. S. Kim, L. R. Dawson, and S. Krishna, Appl. Phys. Lett. 91, 263504 (2007).

${ }^{11}$ E. K. Huang, A. Haddadi, G. Chen, B.-M. Nguyen, M.-A. Hoang, R. McClintock, M. Stegall, and M. Razeghi, Opt. Lett. 36, 2560 (2011).

${ }^{12}$ M. Z. Tidrow, X. Jiang, S. S. Li, and K. Bacher, Appl. Phys. Lett. 74, 1335 (1999).

${ }^{13}$ S. Krishna, D. Forman, S. Annamalai, P. Dowd, P. Varangis, T. Tumolillo, A. Gray, J. Zilko, K. Sun, M. Liu, J. Campbell, and D. Carothers, Appl. Phys. Lett. 86, 193501 (2005).

${ }^{14}$ N. Cohen, G. Mizrahi, G. Sarusi, and A. Sa'ar, Infrared Phys. Technol. 47, 43 (2005).

${ }^{15}$ A. Bezinger, H. C. Liu, B. Aslan, M. Buchanan, A. J. Springthorpe, P. J. Poole, D. A. Cardimona, and G. J. Brown, Electron. Lett. 43, 685 (2007).

${ }^{16}$ E. Cho, B. McQuinston, W. Lim, D. Rafol, C. Hanson, R. Nguyen, and A. Hutchinson, Proc. SPIE 5074, 735 (2003).

${ }^{17}$ S. V. Bandara, S. D. Gunapala, D. Z. Ting, J. K. Liu, C. J. Hill, J. M. Mumolo, and S. Keo, Infrared Phys. Technol. 50, 211 (2007).

${ }^{18}$ T. Martin, R. Brubaker, P. Dixon, M.-A. Gagliardi, and T. Sudol, Proc. SPIE 5783, 13 (2005).

${ }^{19}$ H. C. Liu, J. Li, J. R. Thompson, Z. R. Wasilewski, M. Buchanan, and J. G. Simmons, IEEE Electron Dev. Lett. 14, 566 (1993).

${ }^{20}$ L. C. Lenchyshyn, H. C. Liu, M. Buchanan, and Z. R. Wasilewski, J. Appl. Phys. 79, 8091 (1996).

${ }^{21}$ E. H. Steenbergen, M. J. DiNezza, W. H. G. Dettlaff, S. H. Lim, and Y.-H. Zhang, Appl. Phys. Lett. 97, 161111 (2010).

${ }^{22}$ E. H. Steenbergen, M. J. DiNezza, W. H. G. Dettlaff, S. H. Lim, and Y.-H. Zhang, Infrared Phys. Technol. 54, 292 (2011).

${ }^{23}$ O. O. Cellek and Y.-H. Zhang, Proc. SPIE 8268, 8268N (2012).

${ }^{24}$ B. F. Levine, J. Appl. Phys. 74, R1 (1993).

${ }^{25}$ A. Nedelcu, X. Marcadet, O. Huet, and P. Bois, Appl. Phys. Lett. 88, 191113 (2006).

${ }^{26}$ S. D. Gunapala, B. F. Levine, L. Pfeiffer, and K. West, J. Appl. Phys. 69, 6517 (1991).

${ }^{27}$ R. Rehm, H. Schneider, C. Schönbein, and M. Walther, Physica E 7, 124 (2000).

${ }^{28}$ O. O. Cellek and C. Besikci, Semicond. Sci. Tech. 19, 183 (2004). 